\title{
QoS Translation and Admission Control for MPEG Video
}

\author{
Kiwook Kim \\ Computer Science Department \\ University of Ilinois at Urbana-Champaign \\ Klara Nahrstedt \\ Computer Science Department \\ University of Illinois at Urbana-Champaign
}

\begin{abstract}
In this paper we present an integrated view of translation and admission control relations for MPEG video streams between a multimedia distributed application such as video-on-demand, and its underlying system resource, CPU. Both services are presented as core services of our Quality of Service (QoS) communication model. This communication model is analyzed for different MPEG grouping schemes and communication paradigms. We show under these schemes and paradigms the translation and admission control implications for deterministic QoS specification which must be considered in any MPEG-based multimedia system.
\end{abstract}

Keywords

QoS translation, Admission Control, MPEG video, Quality of service

\section{INTRODUCTION}

Communication model for quality-of-service(QoS) guarantees has been proposed to ensure the guaranteed service video servers and clients need [2]. The service user sends the QoS specification to the service provider. The service provider translates the overall, higher-level QoS specification requirements into requirements for individual resources which the service provider needs for service provisions. Once the QoS specification has the corresponding QoS/resource domain representation (e.g. CPU resource domain), the resource broker in the service provider can perform admission control. The admission control either admits the resource amount needed for the corresponding QoS requirement, or rejects the resource amount, or suggests new resource amount, which results in new QoS.

This paper presents detailed analysis of the communication model for QoS guarantees if the service user is a MPEG video application and the service provider is the underlying system layer resource, specially processor.

According to the communication model, we consider application $Q o S$ tailored toward MPEG video and corresponding system QoS parameters. We summarize all considered QoS parameters with their notations in Table 1.

\section{QOS TRANSLATION AND ADMISSION CONTROL FOR CPU} Admission control performs schedulability tests to decide if real time scheduling can be achieved. With respect to schedulability of periodic jobs, Liu and 


\begin{tabular}{clll}
\hline QoS type & QoS parameter & Symbol & Comment \\
\hline \multirow{2}{*}{ Application } & Sample size & $M_{A}$ & \\
QoS & Sample size (I, P, B) & $M_{A}^{I}, M_{A}^{P}, M_{A}^{B}$ & \\
& Sample rate & $R_{A}$ & \\
& Sample rate (I, P, B) & $R_{A}^{I}, R_{A}^{P}, R_{A}^{B}$ & $R_{A}^{k}=\left(G_{k} / G\right) \cdot R_{A}, k=I, P, B$ \\
& Number of frames per GOP & $G$ & \\
& Compression pattern & $G_{I}, G_{P}, G_{B}$ & $G=G_{I}+G_{P}+G_{B}$ \\
& Original size of GOP & $M_{G}$ & $M_{G}=\sum_{k=I, P, B} G_{k} \cdot M_{A}^{k}$ \\
& Processing size of GOP & $M_{G}^{\prime}$ & \\
& Degradation factor & $D$ & $D=\left(M_{G}^{\prime} / M_{G}\right)$ \\
\hline System & Computation time & $C\left(C_{F}, C_{G}, C_{M}\right)$ & \\
QoS & Cycle time & $T\left(T_{F}, T_{G}\right)$ & \\
\hline
\end{tabular}

Table 1 QoS specification

(a)

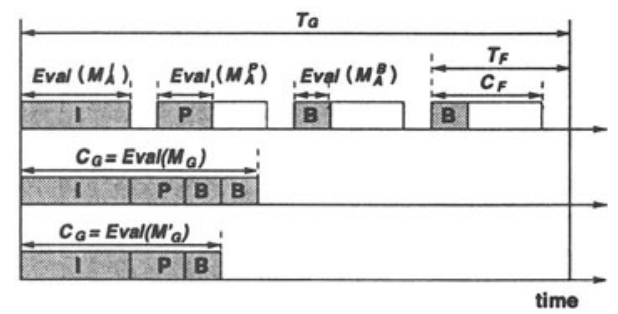

Figure 1 Frame-based scheme and GOP-based scheme

Layland [1] suggested rate monotonic(RM) scheduling and earliest deadline first(EDF) scheduling based on specific assumptions. Both of them require as parameters computation time $(C)$ and cycle time $(T)$ for their schedulability test.

Frame-based scheme In frame-based scheme, the processor allocates scheduling time to process and transmit each frame. In terms of scheduling, RM and EDF have the assumption that execution times need to be constant for all periodic jobs. In order to fulfill this assumption, maximal computation time for all processing units is allocated to process each unit. The maximal computation time is the time required to process maximal size of I frame in MPEG video as shown in Figure 1a, so the computation time is as follows.

$C_{F}=\max \left(\operatorname{Eval}\left(M_{A}^{I}\right), \operatorname{Eval}\left(M_{A}^{P}\right), \operatorname{Eval}\left(M_{A}^{B}\right)\right)=\operatorname{Eval}\left(M_{A}^{I}\right)$

where $\operatorname{Eval}\left(M_{A}^{I}\right) \gg \operatorname{Eval}\left(M_{A}^{P}\right) \gg \operatorname{Eval}\left(M_{A}^{B}\right)$. Eval is the function to measure the computation time of a given size of MPEG frame(s). Also, since sample rate $R_{A}$ is known for the MPEG video characteristics (application QoS), the cycle time $T$ can be computed as $T_{F}=\frac{1}{R_{A}}$.

The frame-based scheme is adequate to apply to a MPEG compressed video which has relatively small range of variation in any frame size. This is the case if MPEG stream consists of only consecutive I frames, not including $P$ and $B$ frames.

This scheme has notable drawbacks for scheduling of communication tasks if MPEG video has compression pattern including $P$ and $B$ frames. The reason is that the processor is underutilized because $P$ and $B$ frames use up only 
a portion of allocated schedule time. Also, since each frame is individually packetized, processed, and transmitted, these steps cause increased overheads in proportion to the number of frames. The extra time for context switching is the typical overhead belonging to this case.

GOP-based scheme A GOP includes at least one I frame to be a reference frame along with $\mathrm{P}$ and $\mathrm{B}$ frames. Each GOP is independent of the other GOP's, since all the frames in a GOP can be decoded without referring to other GOP's. In this respect, a GOP can be a processing unit of MPEG video data. Using definition in QoS specification, the computation time needed to process a GOP is

$C_{G}=\operatorname{Eval}\left(M_{G}\right)=G_{I} \cdot \operatorname{Eval}\left(M_{G}^{I}\right)+G_{P} \cdot \operatorname{Eval}\left(M_{G}^{P}\right)+G_{B} \cdot \operatorname{Eval}\left(M_{G}^{B}\right)$

and the cycle time to process a GOP corresponds to $T_{G}=\frac{G}{R_{A}}$. Here we find the fact that computation time for all frames in a GOP has larger value in frame-based scheme than in GOP-based scheme, since

$$
C_{G}<\left(G_{I}+G_{P}+G_{B}\right) \cdot \operatorname{Eval}\left(M_{G}^{I}\right)=G \cdot C_{F}
$$

The above computation and cycle time is in the case that all the frames in a GOP are processed and sent to clients. On occasion, such a full service cannot or does not need to be provided. For example, when the server does not have enough scheduling time to support full service, it could suggest degraded service to a client by sending a part of GOP. Also the server does not need to send full video frames if client does not want to get full delivery service. All these range of services can be represented using degradation factor, $D=\frac{M_{G}^{\prime}}{M_{G}}$, of which value ranges $\left[\frac{M_{A}^{I}}{M_{G}}, \frac{M_{G}}{M_{G}}(=1)\right]$, since $M_{G}^{\prime}$ could take its value as one I frame as the lowest bound, or all frames in GOP as the upper bound. Once we define $D$, the computation time is

$C_{G}=\operatorname{Eval}\left(M_{G}^{\prime}\right)=\operatorname{Eval}\left(M_{G} \cdot D\right)$

Figure 1b illustrates a full service in which the server processes a whole GOP that comprises I, P, and two B frames. As a degraded service, one of B frames is dropped out, so it is not processed or transmitted in Figure 1c. In this case $D$ is smaller than 1 , because $M_{G}^{\prime}$ corresponds to time needed to compute a GOP excluding a B frame.

This scheme has advantages over frame-based scheme for communication tasks. First, this scheme results in maximal CPU utilization. Secondly, various services can be specified by both server and client using degradation factor. Third, this scheme has less overheads for packetizing, processing and transmitting compared to frame-based scheme. But if the loss of a GOP packet occurs in a network, all frames in a GOP are lost.

Multicasting scheme We now consider a server which receives a number of requests for the same video object. There can be two different schemes to process these requests on the video server or router: unicasting and multicasting. Unicasting scheme means that all demands on video data is processed separately, even though clients request the same video data simultaneously. On the other hand, multicasting scheme deals with simultaneous demands on 
the same video data as one demands. As a result, there exists only one I/O stream from disk to CPU to support those requests.

We can relax the assumption of simultaneous requests on the same video. If we employ the batching technique, a group of demands on the same video which arrive within specified duration can be processed at the same manner. Let $\Delta t$ denote the specified duration, which can be obtained by heuristics.

Now we present the translation of QoS parameters in the multicasting compared to unicasting scheme. Let $\lambda$ be the arrival rate of requests, and $N$ be a random variable denoting the number of requests. If we assume the arrival process is Poisson and the system is $M / G / 1$, then the expected number of requests is $E[N]=\lambda \cdot \Delta t$ and, expected latency $(L)$ for a request [3] is $E[L]=\frac{\Delta t(2+\lambda \Delta t)}{2(1+\lambda \Delta t)}$. Then the computation time of requests within $\Delta t$ in unicasting scheme becomes

$C_{U}=\sum_{i=1}^{E[N]}\left(E\left[L_{i}\right]+C_{i}\right)+c s$

where $c s$ denotes accumulated context switching time for all requests. When using multicasting scheme, the computation time amounts to

$C_{M}=E\left[L_{i}\right]+C_{i}$

which is the time needed to process only one processing unit.

\section{CONCLUSION}

This paper presented an integrated view of translation between MPEG compressed streams and underlying resource, CPU; and its admission control within the resource management. Both services were presented as core services of communication model. This communication model was analyzed for different representation schemes of MPEG video (frame/GOP based) and for different communication paradigms (unicast/multicast). We showed under these schemes and paradigms translations and admission control implications which must be considered in multimedia systems processing and communicating MPEG video streams.

\section{REFERENCES}

[1] C. Liu and J. Layland. Scheduling Algorithms for Multiprogramming in a Hard Real Time Environment. JACM, 20(1):46-61, 1973.

[2] K. Nahrstedt and J. Smith. Design, Implementation and Experiences of the OMEGA End-Point Architecture. IEEE JSAC, 1996.

[3] H. Takagi. Queueing Analysis: a Foundation of Performance Evaluation, vol 1: Vacation and Priority Systems. North-Holland, New York, NY, 1991.

Kiwook Kim is a graduate student majoring in Computer Science at UIUC. He got his MS and BS degrees in Seoul National University in Korea in 1994 and 1996 respectively.

Klara Nahrstedt is an assistant professor in Computer Science at UIUC. She got her $\mathrm{PhD}$ from University of Pennsylvania in 1995. 